\title{
Horner's syndrome caused by an intercostal chest drain
}

\author{
P CAMPBELL, T NEIL, P N WAKE
}

From the District General Hospital, Warrington

\begin{abstract}
Horner's syndrome occurred in a young woman as a complication of the treatment of a traumatic pneumothorax with an intercostal drain. The nerve damage probably occurred when the lung had fully re-expanded, pressing the tip of the intercostal drain, lying at the apex of the pleural cavity, on to the sympathetic chain.
\end{abstract}

Horner's syndrome, consisting of ipsilateral ptosis, papillary constriction, and enophthalmos following section of the cervical sympathetic chain, is a well recognised complication of several conditions, including apical lung tumours, trauma to the neck, neurological disease, cervical lymphadenopathy, vascular disorders, and various surgical procedures affecting the thoracic inlet. Only five cases of the syndrome resulting from the use of a thoracostomy tube have been recorded.

\section{Case report}

A 21 year old woman was admitted to Warrington District General Hospital after a fall from her motorcycle. She complained of left sided chest pain but no other injuries. Examination suggested a left pneumothorax without evidence of rib fracture. There was no sign of any appreciable head or neck injury and neurological examination showed nothing abnormal. Chest radiography confirmed a left pneumothorax without bony injury. An 18 gauge chest drain was inserted through the sixth intercostal space in the mid axillary line and connected to an underwater seal. The lung was slow to expand owing to a persistent air leak, but by the 10 th day only an apical pneumothorax remained. On the 12th day the patient complained of a drooping of her eyelid and on examination a left sided Horner's syndrome with ptosis, miosis, and anhidrosis was noted. Repeat chest radiography showed a fully expanded left lung with the tip of the chest drain lying at the apex of the pleural cavity (fig 1). The drain was removed and when the patient was reviewed at four weeks the Horner's syndrome had completely resolved.

\section{Discussion}

Oculosympathetic paresis characterised by ipsilateral ptosis, pupillary constriction, and enophthalmos after section of the

Address for reprint requests: Dr P Campbell, Department of Surgery, District General Hospital, Warrington WA5 1QG.

Accepted 6 December 1988 cervical sympathetic chain was originally described by Francois Pourfour du Petit in 1710. In 1852 Claude Bernard observed the additional hemifacial vasodilatation, but it was the independent observation of the relation by the Swiss ophthalmologist Johann Friedrich Horner in 1869 which caused the collection of signs to be known as Horner's syndrome. The condition may result from interruption of the sympathetic pathway anywhere from the hypothalamus to the orbit, although the most common area for injury is the second order preganglionic fibres. ${ }^{1}$ These fibres usually arise in the upper two thoracic cord segments, before leaving the spinal cord via the anterior root and joining the sympathetic chain (fig 2). Some authors report, however, that the cervical sympathetic fibres may arise as low as the fourth thoracic segment.'

The first recorded case of Horner's syndrome complicating the use of a thoracostomy tube was by Roseggar and Fritsch in $1980 .^{2}$ Since then only four further cases have been reported. In three patients the drain was inserted after a thoracic procedure ${ }^{3}$ and in the fourth for a spontaneous pneumothorax. ${ }^{4}$ In all cases the chest radiograph showed the drain tip to be at the apex of the pleural cavity. It is postulated that the position of the tube tip causes repeated trauma to the nerve fibres because respiratory movements of the thorax result in local haematoma formation and pressure ischaemia. Sataline and Kraus, however, reported a case of Horner's syndrome as the presenting symptom of a spontaneous

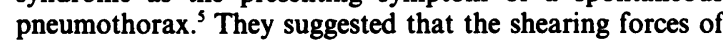
the fascial planes during the development of a pneumo-

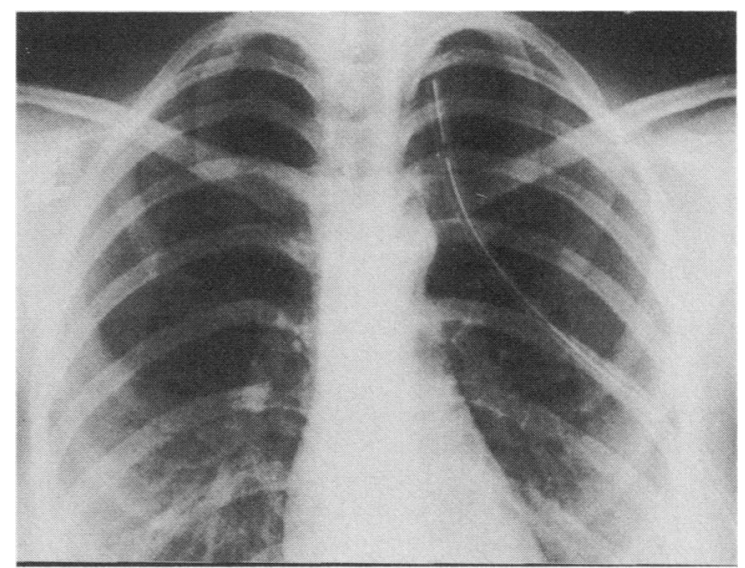

Fig 1 Chest radiograph showing the high position of the tip of the chest drain. 


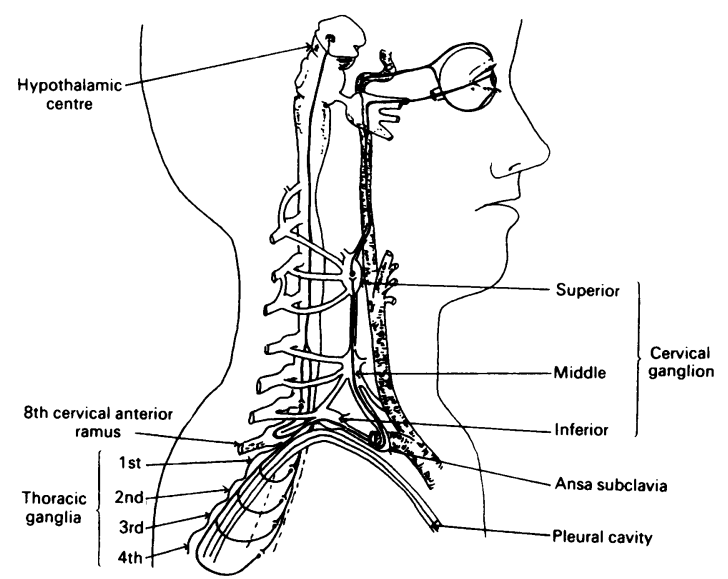

Fig 2 Pathway of the ocular sympathetic fibres.

thorax, whether spontaneous, traumatic, or surgically induced, could cause traction on the sympathetic fibres producing nerve damage. In our patient the fully re- expanded lung could have pressed the drain tip on to the $\underset{x}{x}$ sympathetic chain, producing local ischaemia and neuropraxia.

The outcome of these cases is variable. In our patient the condition resolved within one month, but in only one of the $\overline{0}$ other cases did the patient show complete recovery. In the $\overline{\bar{\omega}}$ other four cases the problems were still present at follow up, $\overparen{\infty}$ which ranged from three months to four years.

These reports emphasise that, although rare, damage to $\mathscr{\aleph}^{2}$ the sympathetic chain from an injudiciously placed intercostal drain may occur and that resolution of the associated. Horner's syndrome cannot be guaranteed.

\section{References}

1 Giles CL, Henderson JW. Horner's syndrome: analysis of 21 cases. Am J Ophthalmol 1958;46:289-96.

2 Roseggar H, Fritsch G. Horner's syndrome after treatment ofw tension pneumothorax with the tube thoracostomy in a new born infant. Eur J Paediatr 1980;133:67-8.

3 Bourque PR, Paulus EM. Chest tube thoracostomy causing Horner's syndrome. Can J Surg 1986;29:202-3.

4 Dutro JA, Phillips LG. Ipsilateral Horner's as a rare complication of tube thoracostomy. N Engl J Med 1985;313:121-2.

5 Sataline LR, Kraus T. Horner's syndrome occurring with spontaneous pneumothorax. $N$ Engl J Med 1965;272:1227-8. 